\title{
LV-VE-2
}

\section{Laparoscopic anatomical S8 segmentectomy by Glissonian approach after dissection of the midplane of the liver}

\author{
Ara CHO, Kwang-Woong LEE*, Su young HONG, Sanggyun SUH, Kwangpyo HONG, Eui Soo HAN, \\ Jeong-Moo LEE, Suk Kyun HONG, YoungRok CHOI, Nam-Joon YI, Kyung-Suk SUH
}

Department of Surgery, Seoul National University College of Medicine, Seoul, Korea

Introduction: Anatomical resection of the liver in HCC is important in terms of oncologic benefit. However, laparoscopic anatomical segment 8 (S8) resection is anatomically complex and technically difficult. Ultrasonography guided dye or ICG injection technique can be a good option, but it is technically demanding and experience dependent.

Methods: A 56-year-old female with underlying chronic hepatitis B virus was diagnosed with $1.8 \mathrm{~cm}$ sized HCC in S8. The tumor was suspected of the microvascular invasion near the middle hepatic vein (MHV) in MRI. Laparoscopic ultrasonography was used to identify the location of mass and the relation to the vasculature. The lower $2 / 3$ of the midplane of the liver was dissected. The branches of MHV draining S4 were divided to get resection margin. After dissection around the right anterior Glissonian pedicle, ligation of S8 pedicle was done. Then, lateral border of S8 was dissected along the ischemic line. MHV was crossed over during the dissection of the upper $1 / 3$ of the midplane of the liver. S8 was anatomically resected.

Results: The operative time was 185 minutes, and the estimated blood loss was $100 \mathrm{~mL}$. The final pathology was HCC and the size of tumor was $18 \mathrm{~mm}$ with $2 \mathrm{~cm}$ safety margin. Follow up CT scan showed congestion in S4 and S5, but no other problems. The patient was discharged on postoperative day 5 without complication.

Conclusions: Laparoscopic anatomical resection of S8 using Glissonian approach after dissection of the midplane of the liver was feasible and it is simpler and easier to apply than dye injection technique. 